\title{
Decision Problems on Unary Probabilistic and Quantum Automata ${ }^{\star}$
}

\author{
Mika HIRVENSALO $^{1, \star \star}$ and Abuzer YAKARYILMAZ ${ }^{2, \star \star \star}$ \\ 1 Department of Mathematics and Statistics, University of Turku, FIN-20014, Turku, Finland \\ ${ }^{2}$ Faculty of Computing, University of Latvia, Raina bulv. 19, Rīga, LV-1586, Latvia \\ mikhirve@utu.fi, abuzer@lu.lv
}

\begin{abstract}
It is well known that the emptiness problem for binary probabilistic automata and so for quantum automata is undecidable. We present the current status of the emptiness problems for unary probabilistic and quantum automata with connections with Skolem's and positivity problems. We also introduce the concept of linear recurrence automata in order to show the connection naturally. Then, we also give possible generalizations of linear recurrence relations and automata on vectors.
\end{abstract}

Keywords: emptiness problem, unary alphabet, probabilistic and quantum automata, Skolem's problem, positivity problem

\section{Introduction}

Finite automata are theoretical models for real-time computing with a finite memory. They form a cornerstone of theoretical computer science, introduced in 1940's and 1950's via a series of papers such as (McCulloch and Pitts, 1943), (Kleene, 1956), (Mealy, 1955), (Moore, 1956), and (Rabin and Scott, 1959). Stochastic versions (probabilistic finite automata (PFAs)) were introduced in (Rabin, 1963), and their properties were extensively studied in (Paz, 1971). In 1997, their quantum versions (quantum finite automata (QFAs)) were introduced in (Moore and Crutchfield, 2000) and (Kondacs and Watrous, 1997) (see (Ambainis and Yakaryılmaz, 2015) for a recent comprehensive survey).

${ }^{\star}$ Some parts of this work was done during Hirvensalo's visit to National Laboratory for Scientific Computing (Brazil) in June 2015 supported by CAPES with grant 88881.030338/2013-01 and during Yakaryllmaz's visit to University of Turku in September 2016.

${ }^{\star \star}$ Partially supported by the Väisälä foundation.

*** Partially supported by CAPES with grant $88881.030338 / 2013-01$ and ERC Advanced Grant MQC. 
It is known that the emptiness (and related) problems are undecidable for PFAs on binary alphabets and since they are generalizations of PFAs, they are also undecidable for QFAs on binary alphabets (Ambainis and Yakaryılmaz, 2015). On unary alphabets, on the other hand, these problems are still open and they are known to be decidable only for some small automata.

In this paper, we present the equivalence of the emptiness problems for unary automata to Skolem's problems and positivity problems for linear recurrence relations (LRR). For this purpose, we also introduce automata versions of LRRs and some of their generalizations defined over vectors.

In the next section, we provide the necessary background. Then, we introduce the concept of linear recurrence automata and their possible generalizations in Section 3. The basic relations on LRRs and generalized finite automata are given in Section 4. The conversions between automata models are listed in Section 5. Finally, we present the status of emptiness problems for the models in Section 6.

\section{Backgrounds}

In this section, we present merely the necessary definitions. For a broader introduction, we refer the reader to (Eilenberg, 1974), (Yu, 1997), and (Paz, 1971) for classical automata, and to (Ambainis and Yakary1lmaz, 2015) for quantum automata.

Throughout the paper, $\Sigma$ denotes the input alphabet not including the right endmarker $\$$, and the extension $\Sigma \cup\{\$\}$ is denoted by $\widetilde{\Sigma}$. Correspondingly, $\widetilde{w}$ represents the string $w \$$ for any $w \in \Sigma^{*}$. Unary alphabet is chosen as $\Sigma=\{a\}$ and the empty string is denoted by $\varepsilon$. For a given machine $M$ and string $w \in \Sigma^{*}, f_{M}(w)$ represents the accepting value/probability of $M$ on $w$. We may also use symbol $\diamond$ to present any of relations in set $\diamond=\{>, \geq,<, \leq,=, \neq\}$.

\subsection{Automata models}

A generalized finite automaton (GFA) $G$ is a 5-tuple

$$
G=\left(Q, \Sigma,\left\{A_{\sigma} \mid \sigma \in \Sigma\right\}, v_{0}, f\right),
$$

where

- $Q=\left\{q_{1}, \ldots, q_{n}\right\}$ is the set of states.

- $A_{\sigma} \in \mathbb{R}^{n \times n}$ is the transition matrix for symbol $\sigma$, i.e. $A[j, i]$ represents the transition value from the $i$-th state to $j$-th state,

- $v_{0}$ is a $|Q|$-dimensional real column vector called initial state, and,

- $f$ is a $|Q|$-dimensional real row vector called final vector.

For a given input $w \in \Sigma^{*}, G$ starts its computation in $v_{0}$, reads $w$ symbol by symbol from left to the right, and, for each symbol, the state vector is updated by multiplying the corresponding transition matrix. That is,

$$
v_{j}=A_{w_{j}} v_{j-1}
$$


where $1 \leq j \leq|w|$. We denote the final state as $v_{f}=v_{|w|}$. The accepting value of $w$ is calculated as

$$
f_{G}(w)=f v_{f}=f A_{w_{|w|}} \cdots A_{w_{1}} v_{0}
$$

which is to say that the automaton $G$ computes a function $\Sigma^{*} \rightarrow \mathbb{R}$. Thus, by picking a real number $\lambda$ called cutpoint, we can split the strings into three different sets: those having accepting value (i) less than $\lambda$, (ii) equal to $\lambda$, and (iii) greater than $\lambda$. Each set or two of them form a language defined by $(G, \lambda)$, i.e $L(G, \diamond \lambda)$ for $\diamond \in \diamond$. More specifically, in the case that $\diamond$ equals to $>$, we have $L(G,>\lambda)=\left\{w \in \Sigma^{*} \mid f_{G}(w)>\right.$ $\lambda\}$, etc.

Moreover, notation $L(G, \lambda)$ refers to the triple $(L(G,<\lambda), L(G,=\lambda), L(G,>\lambda))$ and $L^{+}(G, \lambda)$ refers to the same triple as except that $\varepsilon$ is removed. Remark that for any given pair $(G, \lambda)$ and another cutpoint $\lambda^{\prime}$, there is always another GFA $G^{\prime}$ (that can be designed based on $G$ by adding an additional state) such that

$$
L(G, \lambda)=L\left(G^{\prime}, \lambda^{\prime}\right) .
$$

Therefore, the choice of the cutpoint is not essential unless the number of states is significant.

Let $G$ be a given GFA, $\lambda$ be a cutpoint, and $\diamond \in \diamond$, then determining whether $L(G, \diamond \lambda)=\emptyset$ is an emptiness problem. Remark that the description of $G$ and $\lambda$ should be finite in order to be a "reasonable" computational problem. So, we can formulate the emptiness problem for computable numbers in general and for rational (or integer) numbers in a restricted case. The emptiness problems can be formulated for all the automata models in this paper in a straightforward way, but the special cases are emphasized.

Probabilistic finite automaton (PFA) is a special case of GFA such that only stochastic transition matrices and vectors are allowed. Moreover, a PFA gives its decision based on a subset of states called accepting states. Formally, a PFA $P$ is 5-tuple

$$
P=\left(Q, \widetilde{\Sigma},\left\{A_{\sigma} \mid \sigma \in \widetilde{\Sigma}\right\}, v_{0}, Q_{a}\right),
$$

where, different from a GFA,

- $A_{\sigma}$ is a (left) stochastic transition matrix for symbol $\sigma$, i.e. $A[j . i]$ represents the probability of $P$ going from $i$-th state to $j$-th state after reading symbol $\sigma$,

- $v_{0}$ is a stochastic vector called initial probabilistic state, and,

- $Q_{a} \subseteq Q$ is called the set of accepting states.

For a given input $w \in \Sigma^{*}, P$ starts the computation in $v_{0}$ and reads $w \$$ symbol by symbol in the same way of a GFA:

$$
v_{j}=A_{\widetilde{w}_{j-1}} v_{j-1},
$$

where $1 \leq j \leq|\widetilde{w}|$. The final state is $v_{f}=v_{|\widetilde{w}|}$. The accepting probability of $w$ is calculated as

$$
f_{P}(w)=\sum_{q_{i} \in Q_{a}} v_{f}[i]
$$

The languages recognized by $P$ are defined in a similar way but now $\lambda \in[0,1]$. Any language defined as $L(P,>\lambda)$ (resp. $L(P, \neq \lambda)$ ) is called stochastic (resp. exclusive 
stochastic) (Rabin, 1963) and (Paz, 1971). Moreover, a nondeterministic finite automaton (NFA) can be defined as a PFA $P$ of form $L(P,>0)$. Similarly, a universal finite automaton (UFA) can be defined as a PFA $P$ of form $L(P,=1)$. So, for any PFA $P$, the language $L(P,>0)$ or $L(P,=1)$ (or $L(P,<1)$ or $L(P,=0)$ ) must be regular. On the other hand, any $L(P, \diamond \lambda)$ for some $\lambda \in(0,1)$ and $\diamond \in \diamond$ does not need to be regular.

If each transition matrix of a PFA is double stochastic (both column and row summations are 1), it is called bistochastic PFA (BPFA) (see (Turakainen, 1975)).

A quantum finite automaton (QFA) is a non-trivial generalization of PFA that can be in mixture of quantum states (mixed states) and use superoperators for transitions (Hirvensalo, 2011, Yakary1lmaz and Say, 2011). Formally, a QFA $M$ is a 5-tuple

$$
M=\left(Q, \Sigma,\left\{\mathcal{E}_{\sigma} \mid \sigma \in \widetilde{\Sigma}\right\}, \rho_{0}, Q_{a}\right)
$$

where, different from PFA, $\mathcal{E}_{\sigma}=\left\{E_{\sigma, j} \mid 1 \leq j \leq l_{\sigma}\right\}$ is a superoperator with $l_{\sigma}$ operation elements that is applied when reading symbol $\sigma$ and $\rho_{0}$ is the initial mixed state. For a given input $w \in \Sigma^{*}, M$ starts the computation in $\rho_{0}$, reads $w \$$ symbol by symbol in the same way of a PFA:

$$
\rho_{j}=\mathcal{E}_{\widetilde{w}_{j}}\left(\rho_{j-1}\right)=\sum_{k=1}^{l_{\sigma}} E_{\sigma, k} \rho_{j-1} E_{\sigma, k}^{\dagger}
$$

where $1 \leq j \leq|\widetilde{w}|$. The final state is $\rho_{f}=\rho_{|\widetilde{w}|}$. The accepting probability of $w$ is calculated as

$$
f_{M}(w)=\sum_{q_{i} \in Q_{a}} \operatorname{Tr}\left(\rho_{f}[i, i]\right)
$$

Similar to PFAs, a nondeterministic quantum finite automaton (NQFA) is a QFA $M$ that can define the single language $L(M,>0)$ and a universal quantum finite automaton (UQFA) is a QFA $M$ that can define the single language $L(M,=1)$. On contrary to NFAs and UFAs, NQFAs and UQFAs can define nonregular languages (Yakaryılmaz and Say, 2010).

\subsection{Linear recurrence relations}

A linear recurrence relation (LRR) $u$ with depth $k>0$ (defined over real/complex numbers) is a pair (initial values and coefficients)

$$
u=\left(\left(u_{0}, u_{1}, \ldots, u_{k-1}\right),\left(a_{1}, a_{2}, \ldots, a_{k}\right)\right)
$$

that defines an infinite sequence

$$
u_{0}, u_{1}, \ldots, u_{n}, \ldots,
$$

where $u_{n}=a_{1} u_{n-1}+\cdots+a_{k} u_{n-k}$ for $n \geq k$. 


\subsection{Skolem's problem}

The Skolem's problem (known also as the Pisot problem, (see (Halava et al., 2005) for a general introduction) is to determine whether a given linear recurrence relation

$$
u_{n}=a_{1} u_{n-1}+a_{2} u_{n-2}+\cdots+a_{k} u_{n-k}
$$

over integers with initial values $u_{0}, u_{1}, u_{2}, \ldots, u_{k-1}$, has a member in the sequence with value of $0\left(u_{n}=0\right)$. Number $k$ in the definition is referred as to the depth of the recursion.

Currently it is not known if the Skolem's problem is decidable. The decidability is known for recurrence depths less than 5 , and allegedly for recursion depth 5 (Halava et al., 2005).

The positivity problem is to decide, for a linear recurrent sequence $u_{n}$ over integers, whether $u_{n} \geq 0$ for each $n \geq 1$. The strict positivity problem is to decide whether $u_{n}>0$ for each $n \geq 1$.

Remark that all there problems defined here are defined over integers but they can be trivially extended for real or some specific subsets real numbers.

\section{Linear recurrence automata}

In this section, we introduce automata versions of LRRs and also some of their possible generalizations.

\subsection{Unary versions}

A linear recurrence automaton (LRA) $U=(u, \Sigma)$ with depth $k>0$ is a unary automaton composed by two elements: the LRR $u$ and the unary alphabet $\Sigma$. For each string $a^{j}, U$ assigns $u_{j}$ as the accepting value:

$$
f_{U}\left(a^{j}\right)=u_{j}, \quad j \geq 0 .
$$

Consider the following LRA with depth $k>0$ :

$$
U=(\{a\}, u=(1,0, \ldots, 0),(0, \ldots, 0,1)) .
$$

It is clear that $u_{n}=u_{n-k}$ and the sequence contains only zeros except

$$
u_{0}=u_{k}=u_{2 k}=\cdots=1 .
$$

Therefore, all the following languages are the same and identical to $\operatorname{MOD}_{\mathrm{k}}=\left\{a^{j} \mid j\right.$ $\bmod k \equiv 0\}$ :

$$
L(U,=1), L(U, \geq 1), L(U,>0), L(U, \neq 0) .
$$

A LRR can also be defined over mathematical objects other than numbers. A LRR can be generalized by defining over $\mathbb{R}^{m}$ (or $\left.\mathbb{C}^{m}\right)(m>0)$, called $m$-dimensional LRR over vectors (LRR-V) with depth $k>0$. Formally LRR-V $V$ is a pair

$$
\left.v=\left(\left(v_{0}, v_{1}, \ldots, v_{k-1}\right),\left(A_{1}, \ldots, A_{k}\right)\right\}\right)
$$


that defines an infinite sequence of vectors

$$
v_{0}, v_{1}, \ldots, v_{m}, \ldots
$$

where $v_{n}=A_{1} v_{n-1}+\cdots+A_{k} v_{n-k}$ for $n \geq k$.

The automaton version of an m-dimensional LRR-V with depth $k$ (LRVA) is a triple $V=(v, \Sigma, f)$, where $v$ is a LRR-V and $f \in \mathbb{R}^{m}$ is a row vector. For a given string $a^{j}$ over unary alphabet $\Sigma=\{a\}$, its accepting value is calculated as

$$
f_{V}\left(a^{j}\right)=f \cdot v_{j}
$$

The reader can notice that any $m$-dimensional LRVA with depth 1 is an $m$-state unary GFA. Thus LRVAs can be seen as generalizations of unary GFAs.

The probabilistic version of a LRVA (PLRVA) is a restricted LRVA such that

1. All elements in the sequence including the initial ones are stochastic vectors.

2. Each matrix in $\left\{A_{1}, \ldots, A_{k}\right\}$ is a non-negative multiple of a stochastic matrix providing that the summation $A_{1}+\cdots+A_{k}$ gives a stochastic matrix, i.e. $A_{i}=d_{i} B_{i}$ $\left(d_{i} \geq 0\right)$ for some stochastic matrix $B_{i}$ and $d_{1}+\cdots+d_{m}=1$.

3. Each entry of $f$ is in $[0,1]$.

Thus, the accepting value of each string is in $[0,1]$, which can also be called accepting probability. It is clear that any PLRVA with depth 1 is a unary PFA.

We leave as a future work the definitions of complex and possible quantum versions of LRVAs.

\subsection{Binary versions}

Since LRRs can be seen as unary automata, it is natural to think the binary (or $n$-ary) versions of LRRs and their automata versions.

An LRR defines an infinite sequence. In the binary case, we define an infinite binary tree where each node represents a number (or an object) and so each top to bottom path is an infinite sequence. In order to specify such a sequence, we label each node as a string defined over $\Sigma=\{a, b\}$. The root is empty string. The first level nodes are labeled from left to right as $a$ and $b$. The second level nodes are labeled from left to right as $a a, a b, b a$, and $b b$. In general, if a node is labeled with string $w$, its left child is labeled with $w a$ and its right child is labeled with $w b$.

A tree linear recurrence relation (T-LRR) $t$ with depth $k$ is a triple:

$$
t=\left(\Sigma,\left(t_{w} \mid w \in \Sigma^{\leq k-1}\right),\left(a_{1}, \ldots, a_{k}, b_{1}, \ldots, b_{k}\right)\right),
$$

where $\Sigma$ is a finite alphabet, $\Sigma \leq k-1$ is set of all strings with lengths less than $k, t_{w}$ is the initial value for the node/string $w$ for $w \in \Sigma \leq k-1$, and $\left(a_{1}, \ldots, a_{k}, b_{1}, \ldots, b_{k}\right)$ are coefficients. For any given $w=w_{1} w_{2} \cdots w_{n-k} \cdots w_{n-1} w_{n} \in \Sigma^{*}$ with length $n \geq k$, $t_{w}$ can be calculated as

$$
t_{w}=c_{1} t_{w_{n-1}}+c_{2} t_{w_{n-2}}+\cdots+c_{k} t_{w_{n-k}}
$$


where $c_{i}$ is $a_{i}$ if $w_{n-i+1}$ is symbol $a$ and $c_{i}$ is $b_{i}$ if $w_{n-i+1}$ is symbol $b$.

Any T-LRR can also be seen as an automaton (T-LRA) since its definition includes the alphabet. With depth 1 , we obtain a 1-state GFA, for example. If $|\Sigma|=1$, then we obtain a LRR. The definitions of T-LRR and T-LRA can be extended with some other mathematical objects like vectors, i.e. T-LRR-V and T-LRVA, respectively. Then, we can obtain generalizations of GFAs. In case of probabilistic T-LRA over vectors (TPLRVA), we can assume that each matrix is some non-negative multiple of a stochastic matrix and each vector in the tree is normalized to be stochastic. We can also assume to fix each multiplicative coefficient to $\frac{1}{k}$ for the simplicity, where $k$ is the depth of recursion.

\section{Basic Facts}

Linearly recurrent sequences can be characterized in multiple ways. We give their direct relations with GFAs, and so with PFAs and QFAs.

Proposition 1. For an integer sequence $u_{0}, u_{1}, u_{2}, \ldots$, the following are equivalent:

1. The sequence $u_{n}$ is a linear recurrent sequence.

2. For $n \geq 1, u_{n}=M^{n}[k, 1]$, where $M \in \mathbb{Z}^{k \times k}$ for some $k$.

3. For $n \geq 1, u_{n}=x M^{n} y$, where $x \in \mathbb{Z}^{k}$ is a row vector, $y \in \mathbb{Z}^{k}$ is a column vector, and $M \in \mathbb{Z}^{k \times k}$ for some $k$.

Proof. Implication (1) $\Longrightarrow(2)$ : Assume that a sequence $u_{n}$ is given by first fixing $u_{0}$, $\ldots, u_{k-1}$, and for $n \geq k$ defined by recurrence

$$
u_{n}=a_{k-1} u_{n-1}+\cdots+a_{1} u_{n-k+1}+a_{0} u_{n-k} .
$$

We define

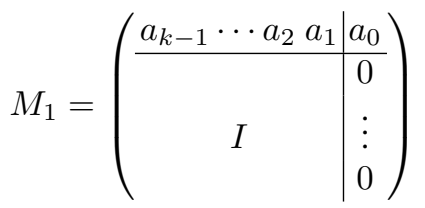

It is easy to see that for each $n \geq 0$,

$$
u_{n}=x M_{1}^{n} y
$$

where $x=\left(\begin{array}{llll}u_{k-1} & \cdots & u_{1} & u_{0}\end{array}\right)$ and $y=\left(\begin{array}{llll}0 & \cdots & 0 & 1\end{array}\right)^{T}$. We denote $\mathbf{0}=\left(\begin{array}{llll}0 & 0 & \cdots & 0\end{array}\right)$ and define a $(k+1) \times(k+1)$-matrix $M$ by

$$
M=\left(\begin{array}{cc}
0 & \mathbf{0}^{T} \\
M_{1} y & M_{1}
\end{array}\right)=\left(\begin{array}{c|c}
0 & \mathbf{0} \\
\hline u_{k} & \\
u_{k-1} & \\
\vdots & M_{1} \\
u_{2} & \\
u_{1} &
\end{array}\right)
$$


Inductively we see that

$$
M^{n}=\left(\begin{array}{cc}
0 & \mathbf{0} \\
M_{1}^{n} x & M_{1}^{n}
\end{array}\right),
$$

and, furthermore, that

$$
\left(M^{n}\right)[k+1,1]=\left(\begin{array}{ll}
0 & y
\end{array}\right)\left(\begin{array}{cc}
0 & \mathbf{0} \\
M_{1}^{n} x & M_{1}^{n}
\end{array}\right)\left(\begin{array}{l}
1 \\
\mathbf{0}
\end{array}\right)=x M_{1}^{n} y=u_{n}
$$

whenever $n \geq 1$.

Implication (2) $\Longrightarrow$ (3) follows directly from equation

$$
M^{n}[k+1,1]=\left(\begin{array}{ll}
0 & y
\end{array}\right) M^{n}\left(\begin{array}{l}
1 \\
\mathbf{0}
\end{array}\right)=u_{n} .
$$

Implication (3) $\Longrightarrow(1)$ : Let $p(x)=x^{k}-a_{k-1} x^{k-1}-\cdots-a_{1} x-a_{0}$ be the characteristic polynomial of matrix $M$. According to the Cayley-Hamilton theorem (Cohn, 1977)

$$
M^{k}=a_{k-1} M^{k-1}+\ldots+a_{1} M+a_{0} I,
$$

and consequently

$$
M^{n}=a_{k-1} M^{n-1}+\cdots+a_{1} M^{n-k+1}+a_{0} M^{n-k}
$$

for any $n \geq k$. It follows that

$$
x M^{n} y=a_{k-1} x M^{n-1} y+\cdots+a_{1} x M^{n-k+1} y+a_{0} x M^{n-k} y,
$$

which is to say that

$$
u_{n}=a_{k-1} u_{n-1}+\cdots+a_{1} u_{n-k+1}+a_{0} u_{n-k},
$$

so (2) is the desired recurrence.

Corollary 1. Let $u_{n}$ be a linear integer recurrent sequence with depth $k>0$. Then, there exists a $(k+1)$-state unary integer GFA $G$ such that

$$
u_{n}=f_{G}\left(a^{n}\right)
$$

for $n \geq 1$.

Corollary 2. Let $G$ be a $k$-state unary integer GFA. Then, there exists a linear (possibly not integer) recurrent sequence $u_{n}$ with depth $k$ such that

$$
u_{n}=f_{G}\left(a^{n}\right)
$$

for $n \geq 0$, where $k>0$.

Proposition 2. Any constant sequence is a linear recurrent sequence. Moreover, if $u_{n}$ and $v_{n}$ are linear recurrent sequences, so are $u_{n} v_{n}$ and $u_{n}+v_{n}$. 
Proof. The claim for constant sequences is trivial. Using Proposition 1, we can write $u_{n}=x_{1} M_{1}^{n} y_{1}$, and $v_{n}=x_{2} M_{2}^{n} y_{2}$. Now $u_{n} v_{n}=\left(x_{1} \otimes x_{2}\right)\left(M_{1} \otimes M_{2}\right)^{n}\left(y_{1} \otimes y_{2}\right)$ (tensor product construction) and $u_{n}+v_{n}=\left(x_{1} \oplus x_{2}\right)\left(M_{1} \oplus M_{2}\right)^{n}\left(y_{1} \oplus y_{2}\right)$ (direct sum construction), and Proposition 1 implies that these sequences are linear recurrent.

Proposition 3. Skolem's problem is reducible to the positivity problem and the strict positivity problem. The strict positivity problem is reducible to the positivity problem.

Proof. Given a linear recurrent sequence $u_{n}$ over integers, deciding whether $u_{n}=0$ for some $n$ is equivalent to deciding whether $u_{n} \neq 0$ for all $n$, which is equivalent to (i) deciding whether $u_{n}^{2}>0$ for all $n$ and (ii) deciding whether $u_{n}^{2}-1 \geq 0$. Thus, Skolem's problem is reducible to (strict) positivity problem.

Given a linear recurrent sequence $u_{n}$ over integers, deciding whether $u_{n} \geq 0$ (resp., $u_{n}>0$ ) for all $n$ is equivalent to deciding $u_{n}+1>0$ (resp., $u_{n}-1 \geq 0$ ) for all $n$. Thus strict positivity problem and positivity problem are reducible to each other.

The reductions between the positivity problem and the strict positivity problem can also be followed over rational numbers but we do not know whether they are reducible to each other over real numbers.

Remark 1. Positivity problem is known to be decidable for linear recurrences with depth at most 5 (Ouaknine and Worrell, 2014b). In addition, the positivity problem for simple recurrences (those having no multiple roots) is known to be decidable for recursion depth at most 9 (Ouaknine and Worrell, 2014a). The ultimate positivity problem is decidable for simple linear recurrences of any order (Ouaknine and Worrell, 2014c).

\section{Conversions between unary automata models}

Conversions between unary automata models are given below (see (Turakainen, 1975), (Hirvensalo, 2011), (Yakaryılmaz and Say, 2010), (Yakaryılmaz and Say, 2011), and (Say and Yakaryılmaz, 2014)):

- For any given integer LRA $U=(u, \Sigma)$ with depth $k$, there exists a $(k+1)$-state integer GFA $G$ such that

$$
L^{+}(U, 0)=L^{+}(G, 0) \text {. }
$$

- For any given rational $n$-state GFA $G$ and cutpoint $\lambda \neq 0$, there exist a rational $(n+1)$-state rational $G^{\prime}$ such that

$$
L(G, \lambda)=L\left(G^{\prime}, 0\right)
$$

Moreover, there exists an integer $(n+1)$-state GFA $G^{\prime \prime}$ such that

$$
L(G, \lambda)=L\left(G^{\prime}, 0\right)=L\left(G^{\prime \prime}, 0\right) .
$$

- For any given $n$-state rational GFA $G$, there exist $(n+2)$-state rational PFA $P$ and $(n+2)$-state algebraic number QFA $M$ such that

$$
L(G, 0)=L\left(P, \frac{1}{n+2}\right)=L\left(M, \frac{1}{n+2}\right) .
$$


- For any given $n$-state rational QFA $M$, there exists an $n^{2}$-state rational GFA $G$ such that

$$
L(M, \lambda)=L(G, \lambda) \text { for any } \lambda \in[0,1] .
$$

- For any given QFA $M$, there exists a QFA $M^{\prime}$ such that

$$
L(M, \neq \lambda)=L\left(M^{\prime}, \neq \lambda^{\prime}\right) \text { for any } \lambda, \lambda^{\prime} \in[0,1] .
$$

- For any given $n$-state integer GFA $G$, there exists an integer LRA $U$ with depth $n$ such that

$$
L^{+}(G, \lambda)=L^{+}(U, \lambda) \text { for any } \lambda \in \mathbb{R} .
$$

\section{The status of emptiness problems}

In this section, we list the decidable problems and the problems which are still open with their restricted but decidable cases.

\subsection{Problems}

The decision problems for LRRs can be formulated by LRAs as given below. Here we also reformulate positivity problems as threshold problems in order to define them as emptiness problems.

- Skolem's problem:

- For a given integer LRA $U$, is $L(U,=0)$ empty?

- More generally, is $L(U,=\lambda)$ empty for a given $\lambda \in \mathbb{R}$ ?

- The positivity problem:

- For a given integer LRA $U$, is $L(U, \geq 0)=a^{*}$ or equivalently the complement of $L(U, \geq 0)$ empty or equivalently is $L(U,<0)$ empty?

- Since deciding whether $u_{n}<0$ for some $n$ is equivalent to deciding whether $-u_{n}>0$ for some $n$, we can also equivalently ask whether $L(U,>0)$ is empty.

- More generally, is $L(U,>\lambda)$ empty for a given $\lambda \in \mathbb{R}$ ?

- The strict positivity problem:

- For a given integer LRA $U$, is $L(U,>0)=a^{*}$ or equivalently the complement of $L(U,>0)$ empty or equivalently is $L(U, \leq 0)$ empty?

- Since deciding whether $u_{n} \leq 0$ for some $n$ is equivalent to deciding whether $-u_{n} \geq 0$ for some $n$, we can also equivalently ask whether $L(U, \geq 0)$ is empty.

- More generally, is $L(U, \geq \lambda)$ empty for a given $\lambda \in \mathbb{R}$ ?

- The exclusivity ${ }^{3}$ problem:

- For a given integer LRA $U$, is $L(U, \neq 0)$ empty?

- More generally, is $L(U, \neq \lambda)$ empty for a given $\lambda \in \mathbb{R}$ ?

Due to the conversions given in the previous section, each decision problem given for LRRs and so LRAs has an equivalent emptiness problem for GFAs, PFAs, and QFAs. In the above list, it is sufficient to change LRA $U$ with unary GFA, PFA, or QFA model in order to obtain the corresponding emptiness problem.

However, remark that the value of $\lambda$ can be only in $[0,1]$ for PFAs and QFAs (the cases for the other $\lambda$ values are trivial) and we should also note that their behaviors are different on the borders.

\footnotetext{
${ }^{3}$ We propose this term inspired by the definition of exclusive stochastic languages.
} 


\subsection{Decidable problems}

PFAs define only regular languages when $\lambda$ is 0 or 1 . So, any emptiness problem in such case is decidable.

The emptiness problem for NQFAs defined with algebraic numbers is known to be decidable (Demirci et al., 2014). Therefore, the problem of whether $L(M,>0)$ or $L(M,<1)$ is empty set for a given QFA $M$ (defined with algebraic numbers) is decidable. Remark that the problem of whether $L(M, \geq 0)$ or $L(M, \leq 1)$ is empty for a given QFA $M$ is trivial.

The remaining cases to determine whether $L(M,=0)$ or $L(M,=1)$ is empty for a given QFA $M$ (the emptiness problem for UQFAs) are still open.

For a given QFA $M, L(M,>0)$ is the same as $L(M, \neq 0)$, and so we can also conclude that the problem of determining whether $L(M, \neq \lambda)$ is empty set is also decidable for any $\lambda \in[0,1]$. Thus, the exclusivity problem is decidable for LRAs, GFAs, PFAs, and QFAs.

\subsection{Open problems}

Now, we can list the open cases:

- Skolem's problem: LRAs, GFAs, PFAs when $\lambda \in(0,1)$, and QFAs when $\lambda \in[0,1]$.

- The positivity problem: LRAs, GFAs, PFAs when $\lambda \in(0,1)$, and QFAs when $\lambda \in(0,1)$.

- The strict positivity problem: LRAs, GFA, PFAs when $\lambda \in(0,1)$, and QFAs when $\lambda \in(0,1)$.

We do not know the answer of any question for any models defined with computable number or any subsets of computable numbers. We know only some decidable results for small automata given in the next subsection.

\subsection{Special cases}

From Remark 1, we know that the positivity problem is decidable for depth 5 . Thus the same problems are decidable also for the following models by using the conversions above:

- 5-state integer GFAs,

- 4-state rational GFAs and also rational PFAs, and,

- 2-state rational QFAs.

\section{References}

Ambainis, A. and Yakaryılmaz, A. (2015). Automata and quantum computing. Technical Report 1507.01988, arXiv.

Cohn, P. M. (1977). Algebra, volume 2. John Wiley and Sons.

Demirci, H. G., Hirvensalo, M., Reinhardt, K., Say, A. C. C., and Yakaryılmaz, A. (2014). Classical and quantum realtime alternating automata. In NCMA, volume 304, pages 101-114. Österreichische Computer Gesellschaft. (arXiv:1407.0334). 
Eilenberg, S. (1974). Automata, Languages, and Machines Volume A. Academic Press, London.

Halava, V., Harju, T., Hirvensalo, M., and Karhumäki, J. (2005). Skolem's problem - on the border between decidability and undecidability. TUCS Technical Reports 683, Turku Centre for Computer Science.

Hirvensalo, M. (2011). Quantum automata theory - a review. In Algebraic Foundations in Computer Science, volume 7020 of LNCS, pages 146-167.

Kleene, S. C. (1956). Representation of events in nerve nets and finite automata. In Automata Studies, pages 3-41. Princeton University Press.

Kondacs, A. and Watrous, J. (1997). On the power of quantum finite state automata. In FOCS'97, pages $66-75$.

McCulloch, W. and Pitts, W. (1943). A logical calculus of the ideas immanent in nervous activity. Bulletin of Mathematical Biophysics, 7:115-133.

Mealy, G. (1955). A method for synthesizing sequential circuits. Bell Systems Technical Journal, 34:1045-1079.

Moore, C. and Crutchfield, J. P. (2000). Quantum automata and quantum grammars. Theoretical Computer Science, 237(1-2):275-306.

Moore, E. (1956). Gedanken-experiments on sequential machines. In Automata Studies, pages 129-153. Princeton University Press.

Ouaknine, J. and Worrell, J. (2014a). On the positivity problem for simple linear recurrence sequences,. In Automata, Languages, and Programming - 41st International Colloquium, ICALP 2014, Part II, volume 8573 of Lecture Notes in Computer Science, pages 318-329. Springer.

Ouaknine, J. and Worrell, J. (2014b). Positivity problems for low-order linear recurrence sequences. In Proceedings of the Twenty-Fifth Annual ACM-SIAM Symposium on Discrete Algorithms (SODA'14), pages 366-379. SIAM.

Ouaknine, J. and Worrell, J. (2014c). Ultimate positivity is decidable for simple linear recurrence sequences. In Automata, Languages, and Programming - 41st International Colloquium, ICALP 2014, Part II, volume 8573 of Lecture Notes in Computer Science, pages 330-341. Springer.

Paz, A. (1971). Introduction to Probabilistic Automata. Academic Press, New York.

Rabin, M. and Scott, D. (1959). Finite automata and their decision problems. IBM Journal of Research and Development, 3:114-125.

Rabin, M. O. (1963). Probabilistic automata. Information and Control, 6:230-243.

Say, A. C. C. and Yakaryllmaz, A. (2014). Quantum finite automata: A modern introduction. In Computing with New Resources, volume 8808 of LNCS, pages 208-222. Springer.

Turakainen, P. (1975). Word-functions of stochastic and pseudo stochastic automata. Annales Academiae Scientiarum Fennicae, Series A. I, Mathematica, 1:27-37.

Yakaryılmaz, A. and Say, A. C. C. (2010). Languages recognized by nondeterministic quantum finite automata. Quantum Information and Computation, 10(9\&10):747-770.

Yakaryılmaz, A. and Say, A. C. C. (2011). Unbounded-error quantum computation with small space bounds. Information and Computation, 279(6):873-892.

Yu, S. (1997). Regular languages. In Rozenberg, G. and Salomaa, A., editors, Handbook of Formal Languages, Vol. 1, pages 41-110. Springer-Verlag New York, Inc.

Received October 5, 2016, accepted November 14, 2016 